\title{
Behavior of Autonomic Nervous System Derived from 1/f Fluctuation Sound Sources in Ultrasonic Range
}

\author{
Hiroki Tada ${ }^{\mathrm{a}}$, Yuta Samaki ${ }^{\mathrm{a}}$, Naruki Shirahama ${ }^{\mathrm{b}}$, Satoshi Watanabe ${ }^{\mathrm{c}}$, Yukio Mori ${ }^{\mathrm{a}}{ }^{*}$ \\ ${ }^{a}$ Salesian Polytechnic, 4-6-8 Oyamagaoka, Machida-shi, Tokyo, 194-0215, Japan \\ ${ }^{\mathrm{b}}$ National Institute of Technology, Kitakyushu College, \\ 5-20-1 Shii, Kokuraminamiku, Kitakyushu-shi, Fukuoka, 802-0985, Japan \\ ${ }^{\mathrm{c}}$ School of Health Sciences at Odawara, International University of Health and Welfare, \\ 1-2-25 Shiroyama, Odawara-shi, Kanagawa, 250-8588, Japan \\ *Corresponding Author: mori@salesio-sp.ac.jp
}

\begin{abstract}
This study has investigated effects of hearing $1 / f$ fluctuation sound source on the automatic nerve activity (the parasympathetic nervous system). At the first stage, we generated an ultrasonic sound with the $1 / f$ fluctuation based on the sinusoidal wave (frequency: $40 \mathrm{kHz}$ ) in order to eliminate preference, which leads to finding out unique effect of $1 / f$ fluctuation. At the second stage, the experiment was conducted to have the subjects hear the sound source in the condition of "non-modulation" or "modulation of $1 / f$ fluctuation". As a result, it was verified that sound source modulated with the $1 / f$ fluctuation affects the autonomic nerve activity of the subjects.
\end{abstract}

Keywords: $1 / f$ fluctuation, LF/HF, ultrasonic sound, autonomic nerve activity.

\section{Introduction}

In modern society humans suffer some sort of external damage caused by mental and physical stresses, etc., which contributes to disorder of the autonomic nerve activity. As proved in the field of human biology, parasympathetic nervous system works predominantly as a method for normalizing the disorder ${ }^{(1)}$. On the other hand, it is known that $1 / f$ fluctuation has a positive effect on relaxing humans. This mental mechanism has been identified in several researchers.

Under circumstances, we infer that the mechanism has the effect of working the parasympathetic nerve activity predominantly. We observed the behavior of autonomic nerves when the subject heard $1 / f$ fluctuation signal in an audible range. As a result, the signal indicated the effect of quieting sympathetic nerves predominantly ${ }^{(2)}$.

However, the result also showed that the behavior of autonomic nerves is affected by the difference in music preference. Consequently, the effect of $1 / f$ fluctuation cannot be unraveled at the moment. In this research, the influence of music preferences needs to be removed so as to identify the effect of $1 / f$ fluctuation.

Thus, we propose that the subject is exposed to $1 / f$ fluctuation signal in an ultrasonic range. In this process, sinusoidal wave at high frequency generated and modulated with $1 / f$ fluctuation signal. For this research, we will construct the measurement system with the DSP (Digital Signal Processor). Then we will investigate the effect of $1 / f$ fluctuation on humans, and measure the LF/HF which is an index of the sympathetic nerve activity. The ultimate purpose of this paper is to reveal an original effect of $1 / f$ fluctuation. As a result, it is shown that the $1 / f$ fluctuation affects the autonomic nerve activity of the subjects, significantly.

\section{Construction of the determining system}

Currently, the generator of $1 / f$ fluctuation signal in the ultrasonic range is not commercially available. Therefore, it is necessary to create a generator. In this section, we describe the determining system we constructed.

\subsection{Generation of the $1 / f$ fluctuation}

The fluctuation is defined as an irregular change from 
a spatial or temporal average of a certain amount. One way to show characteristics of the fluctuations is a power spectrum analysis. In the power spectrum, the fluctuation power of which is in inverse proportion to frequency is called the $1 / f$ fluctuation. In the log-log graph, inclination $\lambda$ of the regression line on the power spectrum is equal to -1 . In general, inclination $\lambda$ of the fluctuation equivalent to white noise is equal to zero ${ }^{(3)}$.

In this research, it is necessary to generate a signal with the $1 / f$ fluctuation characteristic. The method adopting numerical integration is used to conduct this process. The algorithm is shown in the following way ${ }^{(4)}$.

The $n$-times integration of the white noise sequence $x_{m}$ at arbitrary time $m$ can be expressed as follows,

$$
\Delta^{-n} x_{m}=\delta^{n} \sum_{r=0}^{N}\left[\frac{n(n+1) \cdots(n+(r-1))}{r !}\right] \cdot x_{m-r},
$$

where $\Delta$ is a differential operator, $\delta$ is a sampling period and $N$ is parameter of integral range, respectively. When we take the integral of $1 / 2$ time in the case where the $n$ equals $1 / 2,1 / f$ fluctuation sequence $y_{m}$ is shown as following.

$$
\begin{aligned}
& y_{m}=\Delta^{-\frac{1}{2}} x_{m} \\
& =\delta^{\frac{1}{2}}\left[x_{m}+\left\{\frac{1}{(2 \cdot 1 !)}\right\} \cdot x_{m-1}+\left\{\frac{1 \cdot 3}{(4 \cdot 2 !)}\right\} \cdot x_{m-2}+\cdots\right] .
\end{aligned}
$$

Proof is shown to ref.(4).

By the above algorithm, obtained was the sequence of the fluctuation that has the power spectrum as shown in Figure 1. The slope $\lambda$ of the regression line on the power

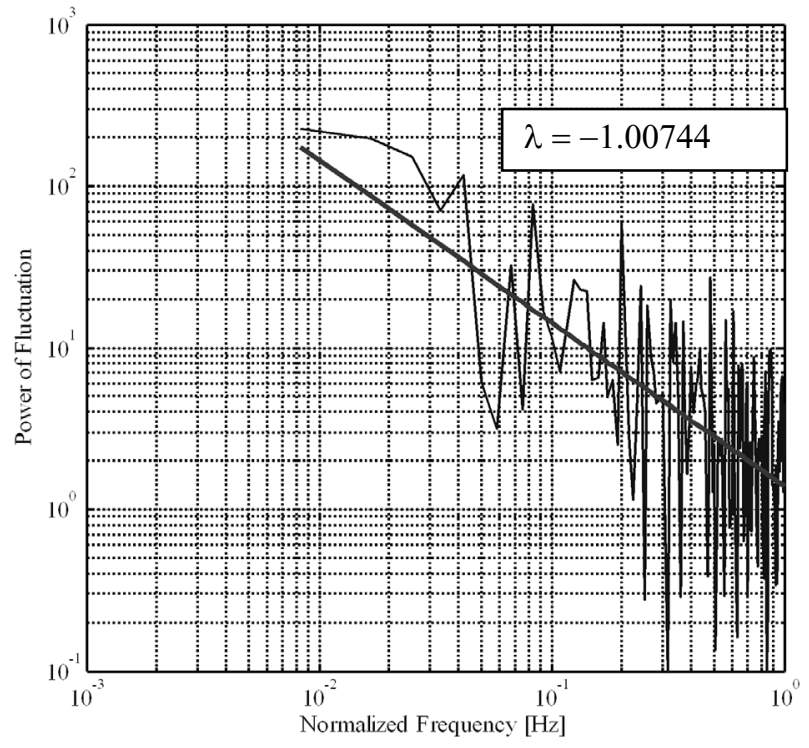

Fig. 1. The power spectrum of generated $1 / f$ fluctuation signal. spectrum was -1.00744 , which demonstrated that is has $1 / f$ fluctuation characteristics. In this research, the $1 / f$ fluctuation signal should be generated by utilizing this sequence. The method will be described in the following section.

\subsection{The modulation and output of the ultrasonic wave}

Figure 2 is an overall view of the system for generating $1 / f$ fluctuation signal in the ultrasonic range. The $1 / f$ fluctuation sequence outputted from the $\mathrm{PC}$ passes through the analog input terminal (CONTEC AI-1608YUSB) inputted into the DSP (TI TMS320C6413DSP). A/D conversion is performed for the $1 / f$ fluctuation in the DSP. In this case, the sinusoidal wave (frequency: $40 \mathrm{kHz}$ ) generated in the DSP is utilized as a carrier signal. The carrier wave is amplitude-modulated with the $1 / f$ fluctuation in the ultrasonic range, as shown in Figure 3. Thus, it transmits information of the $1 / f$ fluctuation signal by using the strength of the amplitude direction. In this process, the

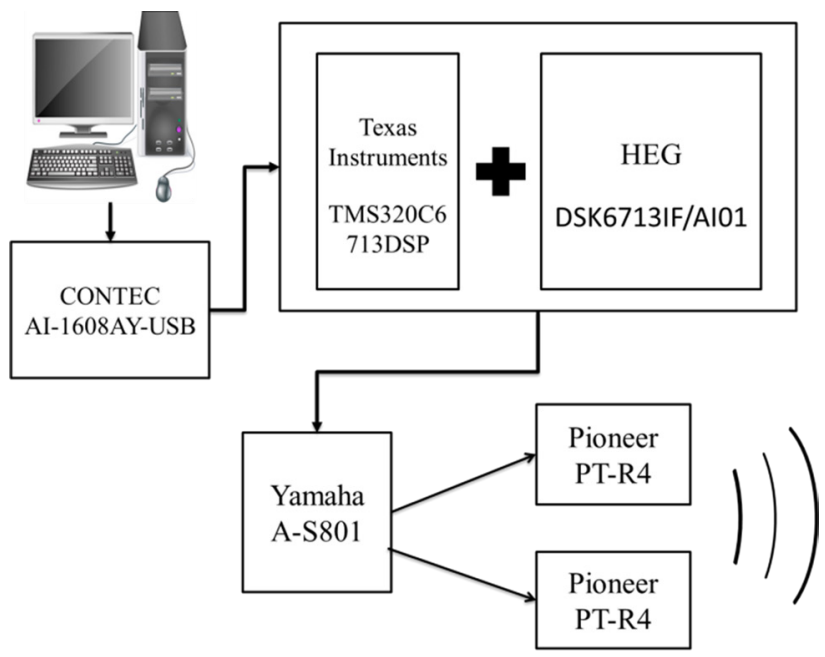

Fig. 2. System of generating $1 / f$ fluctuation in the ultrasonic range.

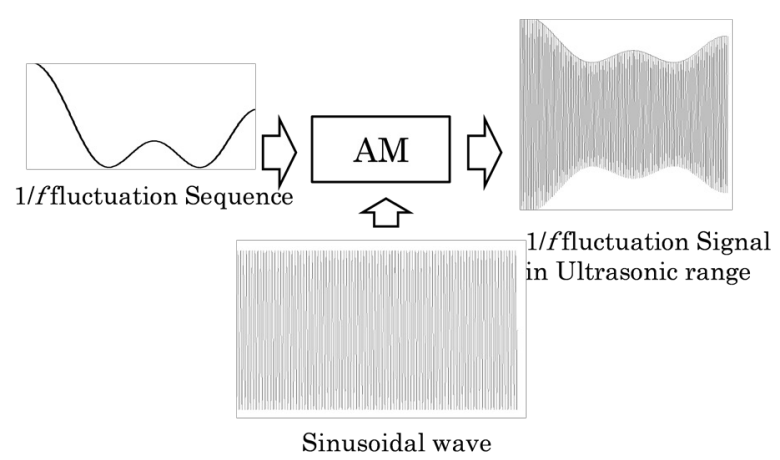

Fig. 3. Amplitude modulation. 
degree of the modulation was $33.3 \%$ for the signal. Furthermore, the DSK (HEG DSK6713IF/AIO1) is used as an extensive interface for DSP. After the signal goes through D/A conversion in the DSP, it is amplified by the amplifier (Yamaha A-S801), which outputs the ultrasonic sound wave by ribbon type super tweeter (Pioneer PT-R4). The tweeter makes it possible to output the ultrasonic wave, which should be heard by the subjects. The subjects hear the ultrasonic sound at its sound pressure level SPL of $100 \mathrm{~dB}$ in the experiment. The pressure level $100 \mathrm{~dB}$ of the ultrasonic sound is large enough even if it is the non-audible sound, which humans are said to feel as a stimulus $^{(5)}$.

However, super-tweeter used in the experiment is mechanically delicate. The pre-experiment was performed to output the ultrasonic sound by the super-tweeter. As a result, the tweeter was broken by an electrical maximum rating before the sound pressure level reached $100 \mathrm{~dB}$, when amplitude was over $2.0 \mathrm{~V}_{\mathrm{pp}}$. Therefore, we decided to use two super tweeters in parallel. Consequently, this allowed us to succeed in dispersing the load on one tweeter into two. The ultrasonic sound outputted from the tweeter was focused on one point by utilizing the ultrasonic directional, and the ultrasonic sound is directed towards the ears of the subjects by tilting the tweeters. The sound pressure level in this condition was measured with the sound level meter. In the Figure 4, the result is that the sound pressure level proves to secure $100 \mathrm{~dB}$ under the condition of non-modulation, when the frequency is $40 \mathrm{kHz}$ shown in Figure 4.

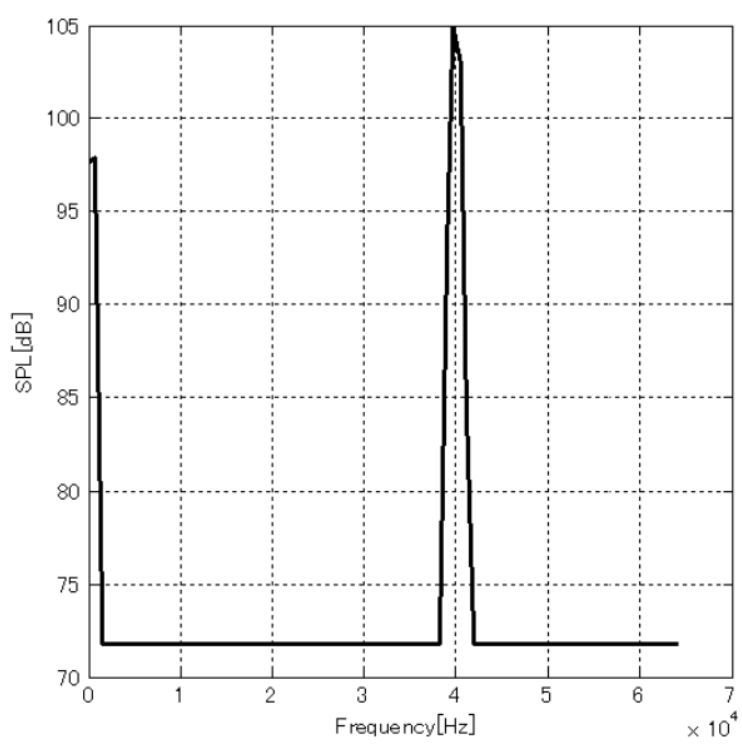

Fig. 4. Sound pressure level (SPL) of ultrasonic sound.

\section{The experiment}

\subsection{Experiment environment in which the ultrasonic sound is heard}

The experiment should be performed under the environment in which it is not impeded by such external disturbance as other noises. Thus, we decided to conduct the experiment in the anechoic chamber as shown in Figure 5.

The subjects are required to take Fowler position (half sitting position) on the adjustable beds in the anechoic chamber. The super tweeter is placed at a distance of $30 \mathrm{~cm}$ from the ears of the subjects, and in this setting the subject hears the $1 / f$ fluctuation sound in the ultrasonic range.

Furthermore, the subject wears the small heart beat sensor (Uniontool WHS-2 as shown in Figure 6) on the chest so as to measure the biomedical signal when the experiment is performed.

Measurements should be performed outside the anechoic chamber, in order to avoid making the subject feel stress or tension. In addition, the ultrasonic modulation system in Figure 2 is installed outside the anechoic chamber, which eliminates the external disturbance caused by noises emitting from such experimental devices as PC.

However, the anechoic chamber creates an unusual setting for humans, so that there was an argument that the chamber itself could be a source of stress. Therefore, the

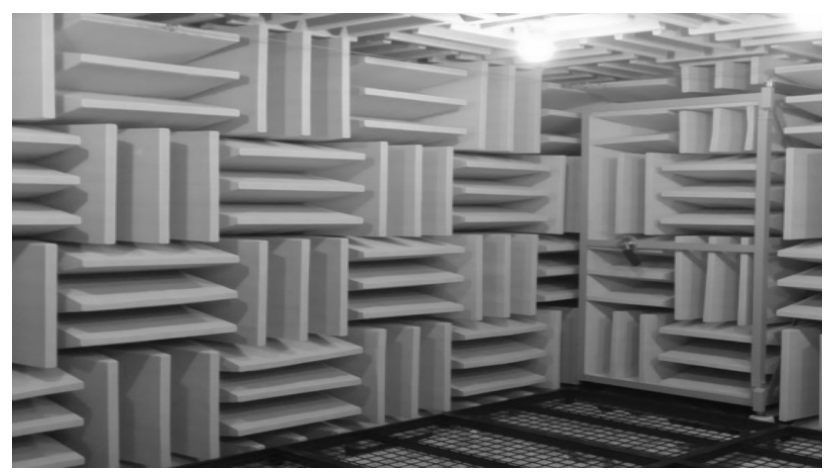

Fig. 5. The anechoic chamber.

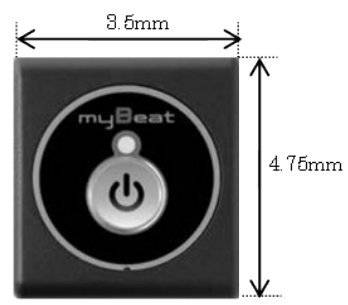

Fig. 6. WHS-2. 
LF/HF was measured for first 4 sections in the experimental procedure of Figure 7 without directing the ultrasonic sound to the subject. If the anechoic chamber is a source of stress, the result should indicate that the value of LF/HF is increased as time proceeds. In fact, the median value showed 1.65, 1.42, 1.15 and 1.20 in a chronological order, which proved to be constant. Therefore, it can be said that there is little possibility that the anechoic chamber becomes a source of stress for the subjects. Consequently, it is less likely that the anechoic chamber itself affects the measurement result.

\subsection{Measurement of the biological signal}

In this research, the biological signal needs to be measured so as to investigate the impact and effect of the $1 / f$ fluctuation sound in the ultrasonic range on humans. Therefore, we focused on the LF/HF that is an indicator describing the sympathetic nerve activity. The larger the value of $\mathrm{LF} / \mathrm{HF}$ index becomes, the most predominant the sympathetic nerve activity becomes. Conversely, the smaller the value of the LF/HF index becomes, the most predominant the parasympathetic nerve activity becomes. The LF/HF index is calculated from the HRV (Heart Rate Variability). The HRV is created as the power spectrum when the horizontal axis is "frequency", and the vertical axis is "power". In this case, it is divided into the low frequency component (LF band $: 0.04 \mathrm{~Hz} \sim 0.15 \mathrm{~Hz}$ ) and the high frequency component ( $\mathrm{HF}$ band $: 0.15 \mathrm{~Hz} \sim 0.4 \mathrm{~Hz}$ ) in the power spectrum. The LF band includes both the sympathetic nerve factor and the parasympathetic nerve factor. In many case, the HF band is as the parasympathetic nerve factor. Therefore, the LF/HF is treated as the indicator for the sympathetic nerve activity ${ }^{(6)}$.

In the actual experiment, the HRV is measured by heart beat sensor that is mounted on the subject's chest,

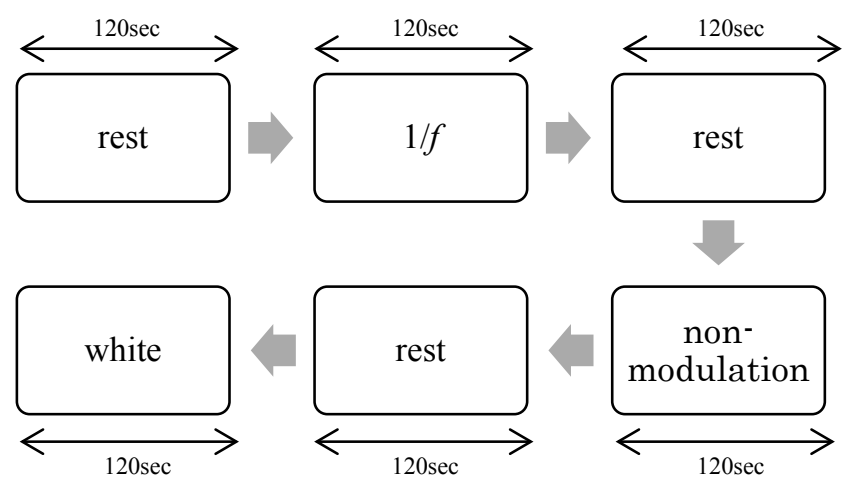

Fig. 7. The experiment procedure. which enables the HRV to calculate the LF/HF index. If the LF/HF value of one section is larger than that of another section, the sympathetic nerve activity becomes predominant. Conversely, if the LF/HF value of one section is smaller than that of another section, the parasympathetic nerve activity becomes predominant.

The measurement data of the HRV transmitted from the heart beat sensor is received in the smartphone through Bluetooth.

\subsection{The experimental procedure}

Figure 7 shows the experiment procedure. The experiment was conducted for 720 seconds in total and 120 seconds in each section. "Rest" section was designated as a section in which the ultrasonic sound was not applied to the subject. "Non-modulation" section was designated as a section in which the non-modulated ultrasonic sound with $40 \mathrm{kHz}$ was applied to the subject. " $1 / f$ " section was designated as a section in which the sinusoidal wave in the ultrasonic range with $40 \mathrm{kHz}$, amplitude-modulated with the 1/f fluctuation, was applied to the subject. "White" section was designated as a section in which the sinusoidal wave in the ultrasonic range with $40 \mathrm{kHz}$, amplitude-modulated with "White noise", was applied to the subject.

The experiment was performed with 30 men and women in their teens or twenties chosen as subjects. All of the subjects declared themselves to be healthy mentally and physically.

\subsection{Data processing}

The LF/HF value is calculated by utilizing the HRV of each measurement section (120 seconds) and compared with one another. In this case, there is a value deviating from the population (outlier) while the LF/HF value shows some variation, In this research, it was determined that the outliners should be detected by adopting Smirnov-Grubbs test ${ }^{(7)}$ under the assumption that the distribution of the data is normal. The research will proceed to the next phase with the data except outliers utilized.

\section{Results and discussions}

Figure 8 shows a histogram indicating the frequencies corresponding to the LF/HF values for each section, which was made up by utilizing the data except outliers. Furthermore, figure 9 shows a box-plot indicating the $\mathrm{LF} / \mathrm{HF}$ values for each section. Here, the box-plot is a graphical representation of variances in the data, the middle 


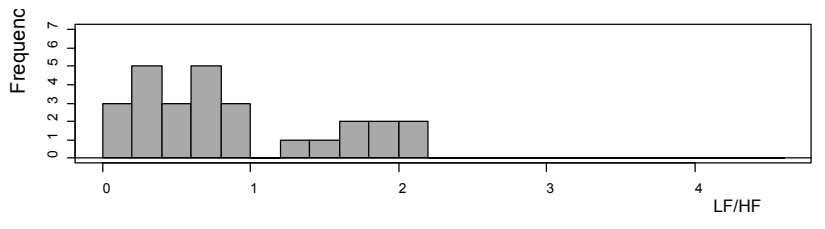

(a) section $=(1)$ non-modulation

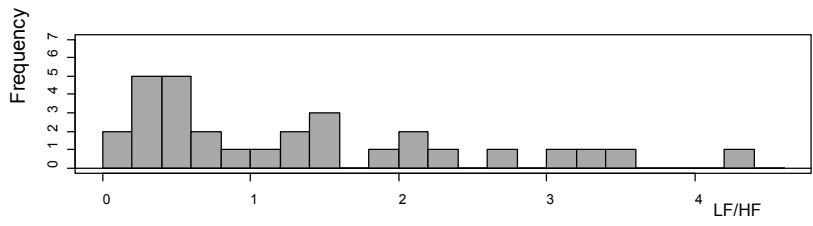

(b) section $=(2) 1 / f$

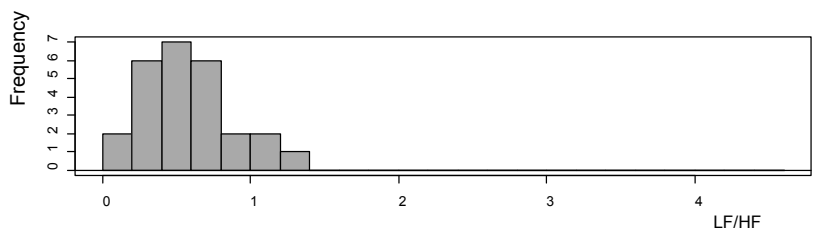

(c) section $=(3)$ white

Fig. 8. This experiment result was shown by utilizing histogram.

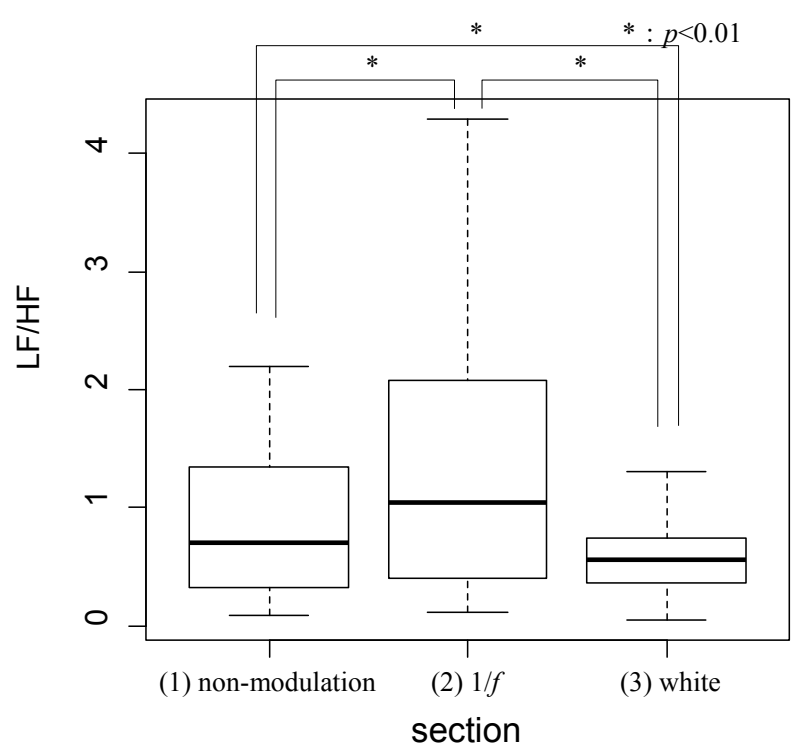

Fig. 9. This experiment result was shown by utilizing box-plot.

thick line is the median, the length of the box is the interquartile range - the upper part is three-quarter value, the lower part is one-quarter value, and the length of the whisker indicates the maximum value (the minimum value) within one and half times the length of the box.

First, the LF/HF values are compared by utilizing the interquartile range value. The interquartile range of the "non-modulation" section is approximately 1.0 , the " $1 / f$ " section is 1.7, and the "white" section is 0.35 . The interquartile range shows the scale of a variance in the values. Therefore, the LF/HF in the " $1 / f$ " section carried the largest variance, and the $\mathrm{LF} / \mathrm{HF}$ in the "White" section carried the smallest variance. Considering this result, we need to examine whether there is a statistically significant point in the variances of these three sections by the statistical test. Therefore, we will adopt a Levene-test ${ }^{(8)}$ to find it. This test identifies the equidistribution of multiple groups of data. Consequently, $p$ values among the respective groups all result in $p<0.01$. The result showed it was recognized that the statistical significance existed in the variances of all sections at a signification level of one percent.

From these results, we found that the value of $\mathrm{LF} / \mathrm{HF}$ in the " $1 / f$ " section data shows larger variance than the other section data. In addition, the variance of the " $1 / f$ " section proved to be different from those of the other sections judging from the result of the Levene test. In the result, it turned out that the effect of the $1 / f$ fluctuation varies from individual to individual. This effect can be considered to be unique to the $1 / f$ fluctuation. Moreover, it is assumed that the main factor for this effect is that the $1 / f$ fluctuation applied did not match the biological rhythm of the subjects. Basically, the biological rhythm has a $1 / f$ fluctuation characteristic ${ }^{(3)}$, and it is inferred that humans get relaxed when given $1 / f$ fluctuation adjusted to their rhythm. Therefore, it is assumed that the parameter of the $1 / f$ fluctuation signal needs to change so as to correspond with the biological rhythm. The examples of the parameter are a change in amplitude of the $1 / f$ fluctuation signal, a change in interval of the sequence, and a change in modulation method etc.

\section{Conclusion}

In this paper, we proposed that the subject is exposed to $1 / f$ fluctuation signal in an ultrasonic range. In this process, sinusoidal wave at high frequency generated and modulated with $1 / f$ fluctuation signal. For this research, we construct the measurement system with the DSP. Then we investigated the effect of $1 / f$ fluctuation on humans, and measure the LF/HF which is an index of the sympathetic nerve activity.

In the chapter 4 , we showed the possibly that $1 / f$ fluctuation sound in ultrasonic range has some effect on a human body. In addition, the effect of the $1 / f$ fluctuation proved to differ from individual to individual, which leads 
to the indication that the effect of $1 / f$ fluctuation can be clarified.

However, it is unknown how the parasympathetic nerve activity should be made predominant, so that further investigation is indispensable. At the same time, the number of subject should be increased.

\section{Acknowledgment}

This work is partially supported by JSPS Grants-in-Aid for Scientific Research, No.16K01890.

\section{References}

(1) L. K. McCorry: "Physiology of the autonomic nervous system.", American journal of pharmaceutical education, Vol. 71, Iss. 4, p. 78, 2007.

(2) S. Watanabe, Y. Matsumoto, M. Tomita and Y. Mori: "Heart rate variability analysis and the subjective evaluation by visual analog scale for subjects listening 1/f fluctuation music" (in Japanese), The Japanese Journal of Biomedical Fuzzy System Association, Vol.15, No.2, pp.1-10, 2013.

(3) T. Musha and M. Yamamoto: " $1 / f$ fluctuation in biological systems", Proceedings 6th International Conference Noise in Physical System, pp. 143-146, 1981.

(4) T. Tobi and K. Toyama: "The trial production to realizing the chaotic-orbit integral calculas-type 1/f-fluctuations", (in Japanese), The Japanese Journal of Biomedical Fuzzy System Association Vol. 7, pp. 92-95, 1994.

(5) K. Ashihara: "Psychoacoustical measurement of hearing threshold for very-high frequency tone", (in Japanese), Acoustical Science and Technology, Vol. 67, No. 5, pp. 208-213, 2011.

(6) A. Malliani, F. Lombardi and M. Pagani: "Power spectrum analysis of heart rate variability: a tool to explore neural regulatory mechanisms", British Heart Journal, Vol. 71, No. 1, pp. 1-2, 1994.

(7) F. E. Grubbs: "Sample criteria for testing outlying observations", Annals of Mathematical Statistics, Vol. 21, No. 1, pp. 27-58, 1950

(8) H. Levene: "Robust tests for equality of variances", Stanford University Press, pp. 278-292, 1960. 